\title{
Biodiversité et écologie des Palparinae du sud du Mali (Neuroptera,
} Myrmeleontidae)

\section{Bruno Michel}

\section{Résumé}

Onze espèces de Palparinae sont signalées du sud du Mali. Palpares obsoletus et $P$. umbrosus sont mentionnées pour la première fois de ce pays. Les complexes gonarcus-paramères de $P$. umbrosus, $P$. nigrescens, $P$. obsoletus et Stenares arenosus sont décrits. Les périodes de vol de plusieurs espèces sont précisées et certaines données concernant leur bioécologie sont apportées.

\section{Abstract}

Biodiversity and ecology of the Palparinae of southern Mali (Neuroptera, Myrmeleontidae). Eleven species of Palparinae are known from southern Mali. Palpares obsoletus and $P$. umbrosus are recorded for first time from this country. The gonarcusparamere complexes of $P$. umbrosus, $P$. nigrescens, $P$. obsoletus and Stenares arenosus are described. The flight periods of several species were determined, and information is given on their bio-ecology.

\section{Citer ce document / Cite this document :}

Michel Bruno. Biodiversité et écologie des Palparinae du sud du Mali (Neuroptera, Myrmeleontidae). In: Bulletin de la Société entomologique de France, volume 104 (1), mars 1999. pp. 45-52;

https://www.persee.fr/doc/bsef_0037-928x_1999_num_104_1_16544

Fichier pdf généré le 25/09/2019 


\title{
Biodiversité et écologie des Palparinae du sud du Mali (Neuroptera, Myrmeleontidae)
}

\author{
par Bruno MICHEL \\ CIRAD/ER, Centre Régional de Recherche Agronomique, B.P. 16, Sikasso, Mali
}

\begin{abstract}
Résumé. - Onze espèces de Palparinae sont signalées du sud du Mali. Palpares obsoletus et $P$. umbrosus sont mentionnées pour la première fois de ce pays. Les complexes gonarcus-paramères de $P$. umbrosus, $P$. nigrescens, $P$. obsoletus et Stenares arenosus sont décrits. Les périodes de vol de plusieurs espèces sont précisées et certaines données concernant leur bio-écologie sont apportées.

Summary. - Biodiversity and ecology of the Palparinae of southern Mali (Neuroptera, Myrmeleontidae). Eleven species of Palparinae are known from southern Mali. Palpares obsoletus and P. umbrosus are recorded for first time from this country. The gonarcus-paramere complexes of $P$.umbrosus, $P$. nigrescens, $P$. obsoletus and Stenares arenosus are described. The flight periods of several species were determined, and information is given on their bio-ecology.
\end{abstract}

Mots clés. - Neuroptera, Myrmeleontidae, Palparinae, région afrotropicale, Mali.

Les Palparinae de l'Afrique de l'Ouest ont été récemment révisés par ProsT (1995). Ce travail recense 18 espèces pour la sous-région, dont 10 pour le Mali. Comme le fait remarquer cet auteur, la plupart du matériel disponible dans les collections d'Europe ou d'Afrique est ancien et peu fourni. Provenant du Mali, seuls 61 individus, souvent incomplètement étiquetés, ont été retrouvés (PrOST, 1991, 1995), et seulement deux espèces, Palpares furfuraceus et $P$. tessellatus, sont reprćsentćcs par au moins 10 exemplaires. Enfin un unique individu provient de la région de Sikasso, la femelle de $P$. nigrescens capturée en 1900 , sans indication précise de localité ni date de récolte, et qui était jusqu'à présent le seul spécimen de cette espèce connu du Mali.

\section{MÉTHODOLOGIE}

Cette étude, débutée en 1993 , a été surtout menée dans la région de Sikasso $\left(11^{\circ} 21^{\prime} \mathrm{N}\right.$, $05^{\circ} 41^{\prime} \mathrm{W}$ ) où les récoltes ont été effectuées tout au long de l'année. Cinq stations ont été visitées régulièrement : $1^{\circ}$ ) la ville de Sikasso $(\mathbf{S}) ; 2^{\circ}$ ) le plateau qui surplombe cette ville au nord, couvert de cultures entrecoupées de jachères annuelles et de prairies à graminées $(\mathbf{P})$; $3^{\circ}$ ) une prairie à graminées, légèrement en pente orientée nord-sud, située à environ $11 \mathrm{~km}$ au nord de Sikasso en bordure de la piste de Longorola (L); $\left.4^{\circ}\right)$ Natiékoura $\left(11^{\circ} 20^{\prime} \mathrm{N}\right.$, $05^{\circ} 51^{\prime} \mathrm{W}$ ), à une vingtaine de kilomètres à l'ouest de Sikasso, où la prairie à graminées est implantée sur la cuirasse ferrugineuse avec un sol caillouteux $(\mathbf{N}) ; 5^{\circ}$ ) les chutes de Farako $\left(11^{\circ} 12^{\prime} \mathrm{N}, 05^{\circ} 29^{\prime} \mathrm{W}\right)$, à $35 \mathrm{~km}$ à l'est de Sikasso où les prairies à graminées, implantées sur un sol plutôt sablonneux, sont situées en bordure d'une forêt-galerie (F). Ces stations sont désignées dans la suite du texte par les lettres $\mathbf{S}, \mathbf{P}, \mathbf{L}, \mathbf{N}$ et $\mathbf{F}$ respectivement. Les autres stations moins fréquentées et plus distantes de Sikasso sont mentionnées dans le texte.

Sauf indication contraire, les récoltes ont été effectuées à vue dans la journée, le plus souvent en fin d'après-midi. L'ensemble du matériel est conservé dans la collection de l'auteur.

\section{LA RÉGION DE SIKASSO}

La ville de Sikasso est située à $374 \mathrm{~m}$ d'altitude, dans l'extrême sud-est du Mali (fig. 1). La pluviosité annuelle avoisine actuellement $1000 \mathrm{~mm}$. Elle était d'environ $1200 \mathrm{~mm}$ 
jusque dans les années 1970 (L'HOTE \& MAHÉ, 1996). Cette région, au relief légèrement vallonné, est incluse dans le "centre régional d'endémisme soudanien" défini par WHITE (1981, 1986). Elle se caractérise par l'alternance d'une saison sèche de novembre à avril, et d'une saison des pluies de mai à octobre, dont la durée et la répartition des précipitations peuvent présenter des variations importantes suivant les années. En dehors des zones de cultures, elle est recouverte par une forêt claire, plus ou moins dégradée. Ce couvert forestier est entrecoupé de prairies à graminées dans lesquelles se rencontrent la majorité des espèces de Myrmeleontidae de la région.

\section{LISTE COMMENTÉE DES ESPÈCES}

\section{1 - Nosa tigris (Dalman, 1823)}

Stations et dates de récolte. - P: 27.IX.95, $2{ }^{\star}$ (nombreux individus observés dans les graminées le long de la piste d'aviation); $5 . \mathrm{X} .94,1 \delta^{\dagger} ; 6 . \mathrm{X} .94,1 \delta^{\dagger} ; 24 . \mathrm{X} .93,19 ; 26 . \mathrm{X} .93,29 ; 7 . \mathrm{XI} .94,19 ; 2 . \mathrm{XII.94}$,

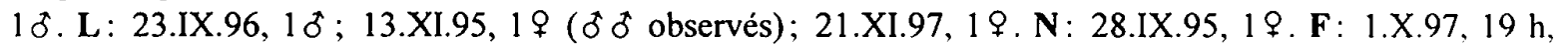

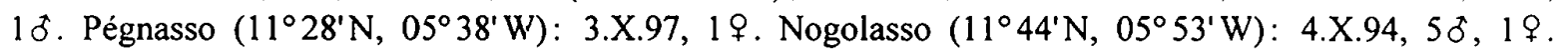
Kourouma $\left(11^{\circ} 53^{\prime} \mathrm{N}, 05^{\circ} 50^{\prime} \mathrm{W}\right)$ : 21.IX.94, $10^{\circ}$. Ména $\left(11^{\circ} 13^{\prime} \mathrm{N}, 06^{\circ} 48^{\prime} \mathrm{W}\right): 18 . I X .96$, entre $16 \mathrm{~h} 30$ et $17 \mathrm{~h}$ $30,2 \delta^{\circ}, 19$. Kadiolo $\left(10^{\circ} 32^{\prime} \mathrm{N}, 05^{\circ} 44^{\prime} \mathrm{W}\right): 22 . \mathrm{X} .96,1 \delta^{\circ}$. Zalogosso $\left(12^{\circ} 44^{\prime} \mathrm{N}, 05^{\circ} 02^{\prime} \mathrm{W}\right): 17 . \mathrm{IX} .96,1 \delta^{\circ}$. Sébékoro $\left(13^{\circ} 00^{\prime} \mathrm{N}, 08^{\circ} 59^{\prime} \mathrm{W}\right): 25 . \mathrm{IX} .96,10^{\circ}, 1$ \% .

Capturé du 17.IX au 2.XII. Très commun dans les formations végétales à graminées.

\section{2 - Palparellus spectrum (Rambur, 1842)}

Stations et dates de récolte. $-\mathbf{L}: 24 . V .95,10,19 ; 25 . V .95,5 \delta^{\star} ; 26 . V .95,19$. N : 27.IV.98, vers

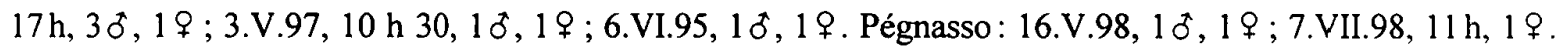
Kourouma: 7.VI.95, $1 \delta^{\circ}, 19$. Bougouni $\left(11^{\circ} 25^{\prime} \mathrm{N}, 07^{\circ} 30^{\prime} \mathrm{W}\right): 11 . \mathrm{V} .98,1 \delta^{\circ}, 2 \delta^{\circ}$.

Capturé du 27.IV au 7.VII. Très commun sur sol dénudé et dans les pelouses naturelles à graminées en début de végétation.

\section{3 - Palpares furfuraceus Rambur, 1842}

Stations et dates de récolte. $-\mathbf{P}: 25 . \mathrm{IX} .97,1 \delta^{\delta} ; 26 . \mathrm{IX} .97$, vers $17 \mathrm{~h} 30,3 \delta^{\star} ; 27 . \mathrm{IX} .95,2 \delta^{\circ} ; 5 . \mathrm{X} .94$, $1 \delta^{*} ; 6 . X .94,6 \delta ; 24 . X .93,19 ; 26 . X .93,2 q ; 14 . X I .96,19 ; 3 . X I I .96,17$ h, 1 \%. F : 1. X.97, 19 h, $1 \delta ;$ 3.X.96, $1 \delta^{\star}$. S : 22.XI.96, $20 \mathrm{~h} 45,19$, piège lumineux. L: 23.IX.97, $2 \delta$. Pégnasso : 3.X.97, $1 \delta^{\star}$. Koumantou $\left(11^{\circ} 25^{\prime} \mathrm{N}, 06^{\circ} 51^{\prime} \mathrm{W}\right)$ : 22.X.97, $1 \delta^{\circ}$. Nogolasso: 4.X.94, $1 \delta^{\circ}, 19$. Kadiolo: 22.X.96, 1 ․ Bamako $\left(12^{\circ} 40^{\prime} \mathrm{N}, 07^{\circ} 59^{\prime} \mathrm{W}\right): 20 . \mathrm{X} .94$, vers $22 \mathrm{~h}, 10^{\circ}, 1 \%$, à la lumière, chassant en vol autour d'un lampadaire (CRES). Sanankoroba $\left(12^{\circ} 23^{\prime} \mathrm{N}\right.$; $\left.07^{\circ} 55^{\prime} \mathrm{W}\right)$ : 13.X.96, $10^{\circ}$. Koulikoro $\left(12^{\circ} 55^{\prime} \mathrm{N}, 07^{\circ} 31^{\prime} \mathrm{W}\right): 4 . \mathrm{XI} .95,1$ \% , sur plateau rocheux. Kokofata $\left(12^{\circ} 53^{\prime} \mathrm{N}, 09^{\circ} 58^{\prime} \mathrm{W}\right): 25 . \mathrm{IX} .96,10^{\circ}$. San $\left(13^{\circ} 17^{\prime} \mathrm{N}, 04^{\circ} 56^{\prime} \mathrm{W}\right): 7 . \mathrm{X} .96,22 \mathrm{~h}$, $1 \delta^{\circ}, 19$, à la lumière, posés contre un mur. Bandiagara $\left(14^{\circ} 21^{\prime} \mathrm{N}, 03^{\circ} 38^{\prime} \mathrm{W}\right): 20.1 \mathrm{XX} .97,11 \mathrm{~h} 30,5 \delta^{\circ}$.

Capturé du 20.IX au 3.XII. Très commun dans les graminées. Attiré par la lumière.

Bandiagara, à $500 \mathrm{~km}$ au nord de Sikasso, est situé dans la zone sahélienne. Dans cette région la pluviosité, de l'ordre de $500 \mathrm{~mm}$ par an, est nettement inférieure à celle de la zone sud.

\section{4-Palpares incommodus (Walker, 1853)}

Stations et dates de récolte. - P: 1.V.97, $1 \delta^{\dagger}$. F: 7.IV.96, 19 h 30, 20; 8.IV.96, 1 \% ; 11.IV.96,

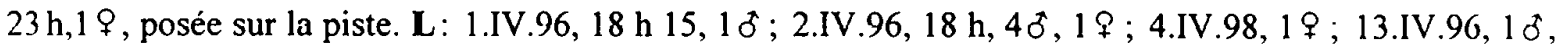
$2 q ; 16 . I V .97,1 \delta ; 3 . V .97,1 q ; 24 . V .95,2 q ; 25 . V .95,3 q ; 26 . V .95,1 \delta ; 28 . V .95,2 \delta$. N: 3.IV.96, $19 ; 7 . I V .98,19$ (1 ठ observé); 17.IV.97, $19 ; 24 . I V .98,17$ h, 1 o ; 22.V.97, $19 ; 6$. VI.95, 1 q. Kadiolo: 6.IV. $96,4 \delta^{\star}, 29$. A $100 \mathrm{~km}$ au nord de Bougouni, direction Bamako: 31.V.98, $11 \mathrm{~h}, 1 \delta^{\star}$.

Capturé du 1.IV au 6.VI. Très commun. Fréquente les mêmes biotopes que $P$. spectrum.

Le 13.IV.96, à Natiékoura, un individu dérangé qui s'envolait vers la cime des arbres a été capturé en vol par un oiseau. 


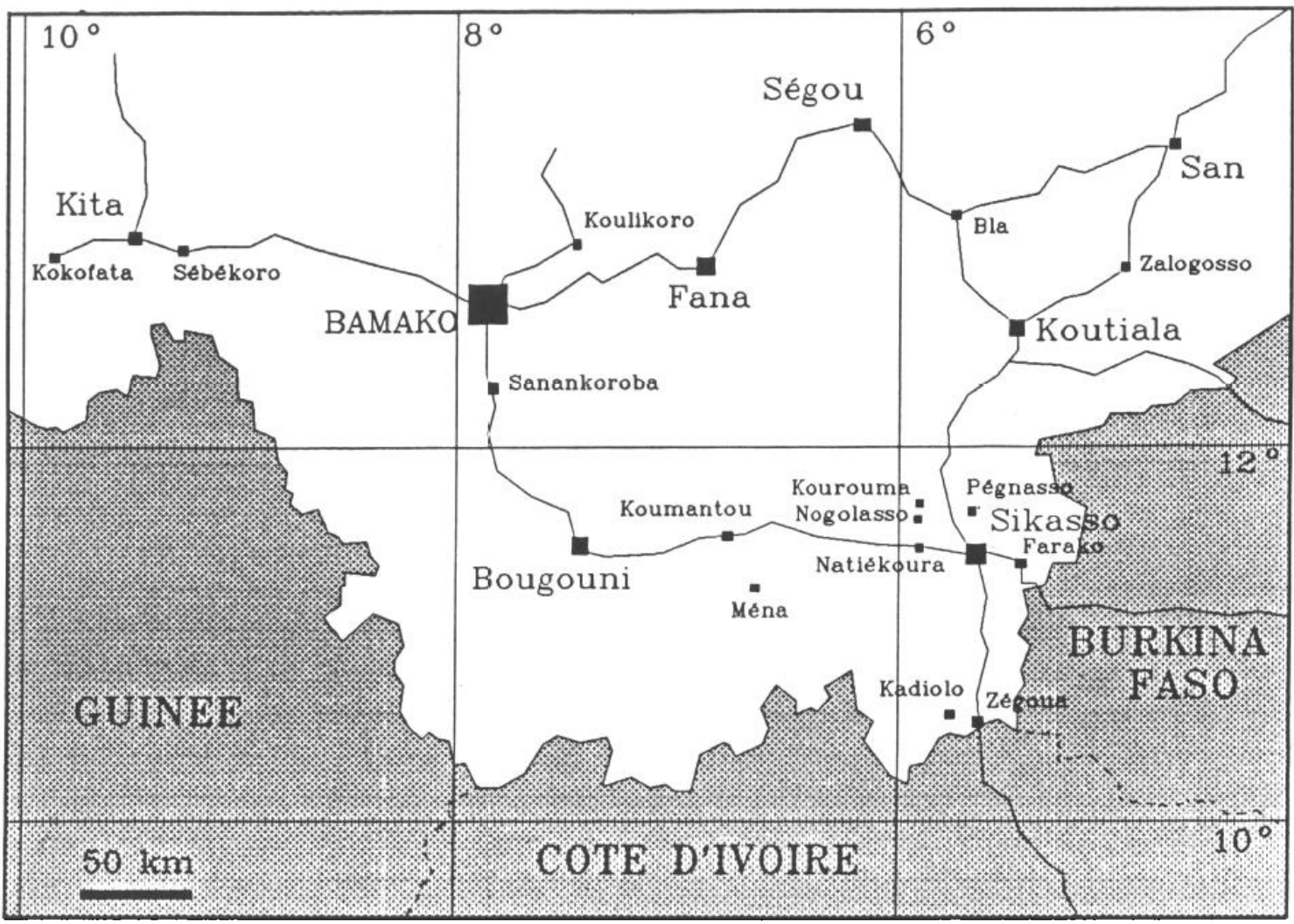

Fig. 1. - Localisation géographique de la région de Sikasso et stations de récolte.

\section{5 - Palpares latipennis Rambur, 1842}

Stations et dates de récolte. $-\mathbf{S}: 30 . X I .96,20 \mathrm{~h} \mathrm{30,1}$, piège lumineux. P: 20.X.97, $2 \delta ; 22 . X .95$, 11 h $30,2 \delta^{\circ} ; 24 . X .93,2 q ; 26 . X .93,2 q ; 29 . X .97,1 \delta^{*}, 1 q ; 3 . X I .94,1 q ; 11 . X I .96,1 q ; 12 . X I .96,1 q ;$ 11.XII.94, $1 q ; 14 . X I I .94,1 q . \mathbf{L}: 24 . X .95,2 \delta, 1 q ; 27 . X .97,1 \delta, 2 q ; 30 . X .95,10 ; 5 . X I I .95,1 q$.

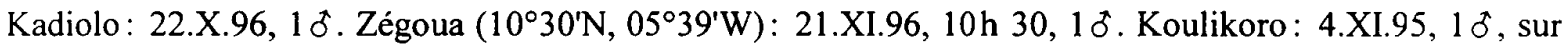
plateau rocheux. Bandiagara: 20.IX.97, $11 \mathrm{~h} 30,60^{\circ}$.

Capturé du 20.IX au 14.XII. Très commun dans les graminées. Attiré par la lumière.

Tout comme $P$. furfuraceus, également capturé en zone sahélienne, à Bandiagara.

\section{6 - Palpares nigrescens Navás, 1913}

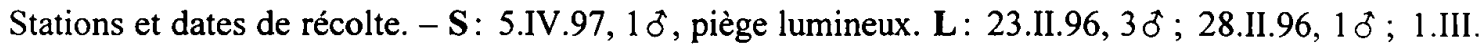

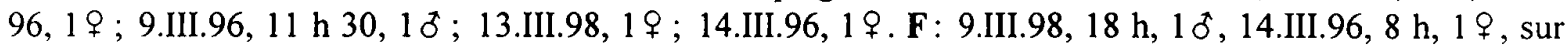
tas de bois; 19.III.96, $1 \%$; 27.III.96, 1q. $\mathbf{N}: 30.1 .96$, $1 \delta ; 10 . I I .97,17 \mathrm{~h}, 29$; 11.II.97, 10,$19 ; 22.11 .97$, $18 \mathrm{~h}, 1 \delta^{\circ}$, posé sur un tronc d'arbre; 4.III.98, 1 \% ; 15.III. 97,$16 ; 18 . \mathrm{III} .96,19 ; 23 . \mathrm{III}$ $.98,1$ \% ; 3.V.97, $10 \mathrm{~h}, 1$ \% .

Capturé du 30.I au 3.V. Jusqu'à présent cette espèce n'était connue du Mali que par l'unique spécimen mentionné en introduction, et les $5 q$

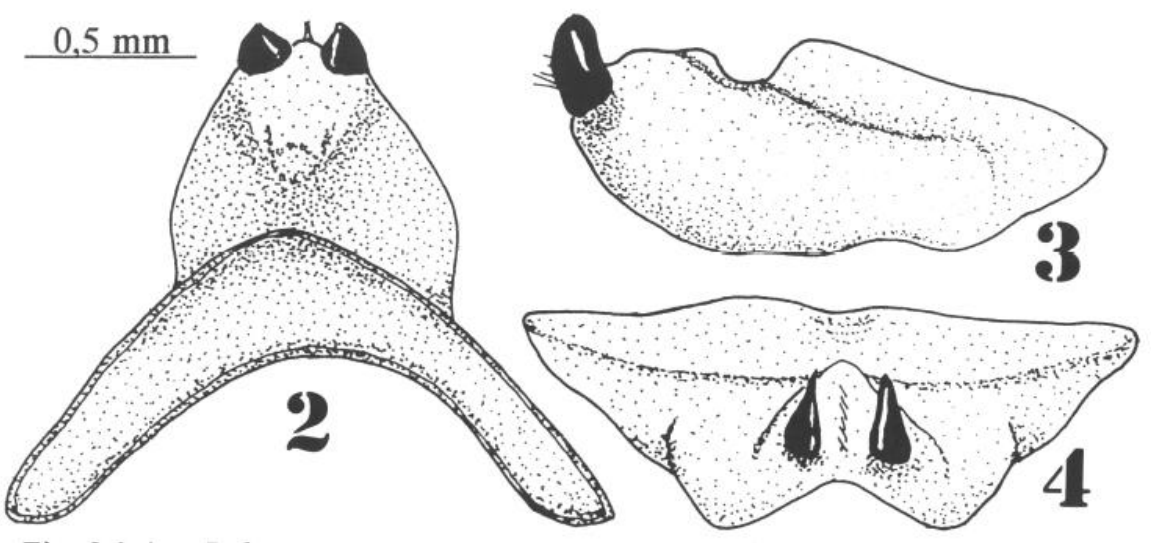

Fig. 2 à 4. - Palpares nigrescens Navás : - 2, Complexe gonarcus-paramères vu de dessus; -3 , Idem, en vue latérale gauche; -4 , Idem, en vue postérieure. 


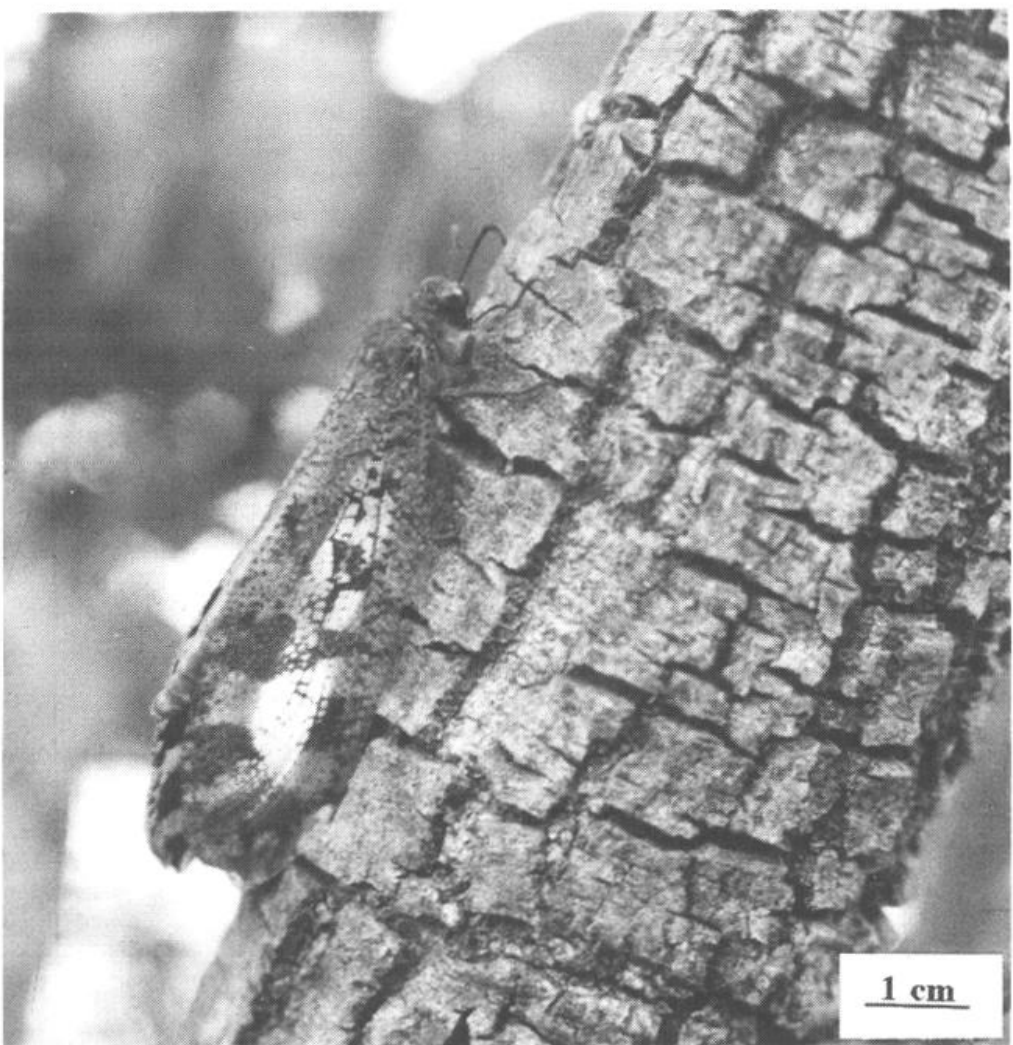

Fig. 5. - Palpares nigrescens Navás, femelle sur une branche. répertoriées d'Afrique de l'Ouest par PROST (1995) ont toutes été capturées en mars. Le seul mâle connu jusqu'ici provenait du Zaïre (Prost, 1995). Nos observations montrent que ce Palparinae est en fait très commun, au moins dans la région de Sikasso, et était considéré comme rare uniquement par manque de prospections appropriées. Attiré par la lumière.

Les paramères, en forme de dent, sont soudés par la base au gonarcus. Ils sont largement séparés et bordent une rangée médiane de fines soies (fig. 2 , $3,4)$.

La plupart des individus s'observent posés sur le sol dépourvu de végétation où leur coloration les rend très difficiles à voir. Ils peuvent également se tenir sur les troncs et les grosses branches des arbres (fig. 5). Lorsqu'il est dérangé, il se réfugie le plus souvent dans le couvert forestier. Ce comportement nous semble quelque peu différent de celui des autres espèces qui ont plutôt tendance à rester dans la formation herbeuse. A trois reprises, des individus que nous avions fait s'envoler, ont été capturés en vol par des oiseaux. Un calao (Tockus sp.) a été observé fin août à Pégnasso tenant un individu dans son bec.

\section{7 - Palpares obsoletus Gerstaecker, 1888}

Station et date de récolte. $-\mathbf{F}: 20 . X .97,19 \mathrm{~h}, 1 \delta^{*}$, piège lumineux.

Espèce "rare" dans la région prospectée, nouvelle pour le Mali. Farako représente la localité la plus au nord, recensée jusqu'à présent pour cette espèce (PROST, 1995).

Les paramères ont un aspect vrillé, avec les bords externes relevés. Présence d'une rangée de soies entre les paramères dont la taille décroît régulièrement de l'avant vers l'arrière (fig. 6, 7).

\section{8 - Palpares radiatus Rambur, 1842}

Station et date de récolte. - Koutiala $\left(12^{\circ} 24^{\prime} \mathrm{N}, 05^{\circ} 28^{\prime} \mathrm{W}\right)$ : 19.VI. $98,6 \mathrm{~h} 30,1 \delta^{\circ}$, posé contre un mur au-dessous d'une lampe, attiré par la lumière au cours la nuit.

Cette espèce n'était connue du Mali que de deux localités situées beaucoup plus au nord: Hombori $\left(15^{\circ} 16^{\prime} \mathrm{N}\right)$ et Dogo $\left(15^{\circ} 10^{\prime} \mathrm{N}\right)$ où on enregistre environ $300 \mathrm{~mm}$ de pluie par an. Koutiala, environ $900 \mathrm{~mm}$ de pluie par an, représente la localité la plus au sud recensée au Mali pour ce Palparinae qui était considéré comme inféodé à la région sahélienne (PROST, 1995). L'autre localité qui semble correspondre à la limite sud de l'aire de répartition de cette espèce est Gaya $\left(11^{\circ} 21^{\prime} \mathrm{N}\right)$ au Niger, qui est situé pratiquement sur le même isohyète que Koutiala. 


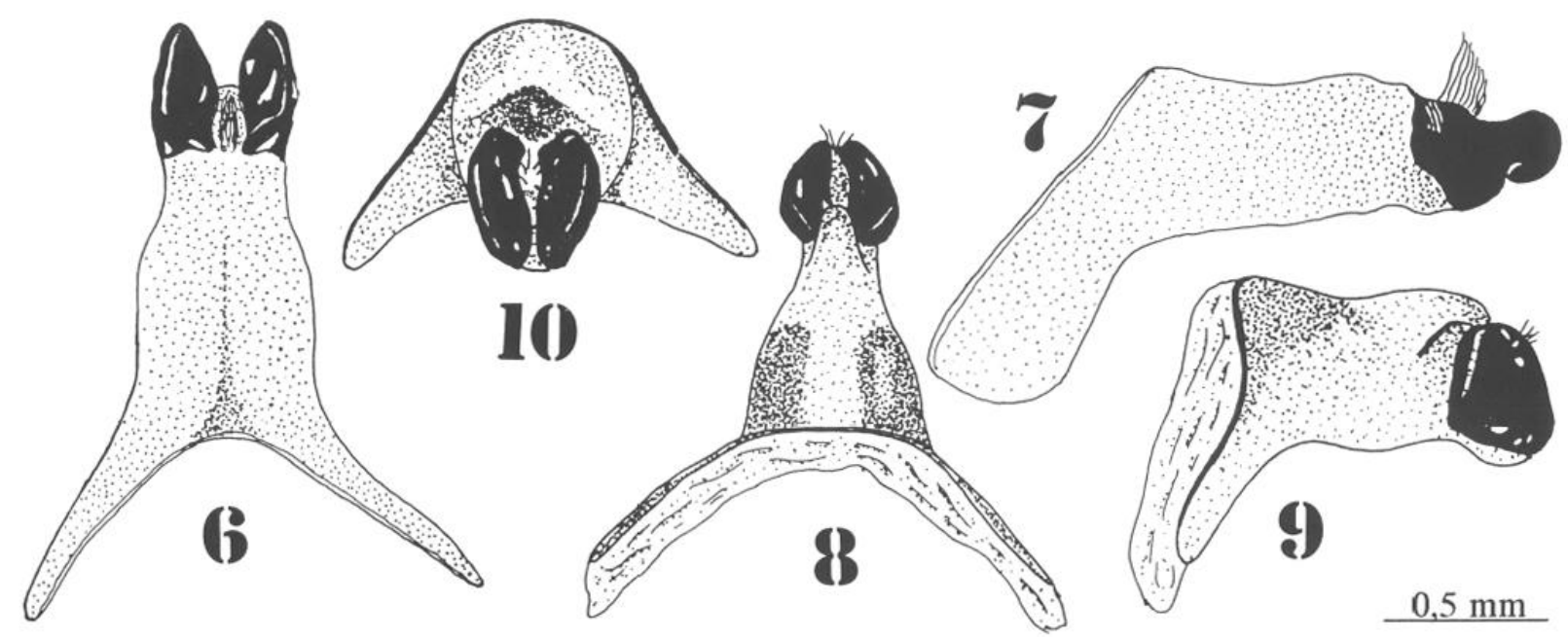

Fig. 6-10. - 6-7, Palpares obsoletus Gerstaecker. -8 à 10, P. umbrosus Kolbe. - 6, 8, Complexe gonarcusparamères, vu de dessus. $-7,9$, Idem, en vue latérale gauche. -10, Idem, en vue postérieure.

9 - Palpares umbrosus Kolbe, 1898

Stations et dates de récolte. - L : 2.V.97, $19 ; 15 . V .97,10$. Pégnasso: $25 . I V .98,1 \delta^{\circ}, 19,12 \mathrm{~h}$; 10.V.97, $1 \delta^{\star} ; 10$. V.98, $10^{*}, 16$ h 45; 14.V.97, $10^{\star}, 3 \% ; 23 . V .98,1 \delta^{\star}, 10$ h. Bougouni : 11.V.98, 19.

Capturé du 25.IV au 23.V. Cette espèce est relativement rare dans la région de Sikasso, et présente une distribution très localisée. Elle n'était connue jusqu'ici que par 5 exemplaires ouest-africains, et est nouvelle pour le Mali (fig. 13).

La région de Sikasso représente pour le moment la limite nord de l'aire d'extension de ce Palparinae.

Tous les individus ont été collectés dans des petits îlots de graminées sèches, situés en bordure de zones arborées ou sous le couvert forestier.

Les paramères sont soudés au gonarcus sur toute leur hauteur. Présence d'une touffe de soies entre les paramères (fig. 8, 9, 10). Le nombre de dents portées par les protubérances des ectoproctes n'est pas constant et varie, chez les exemplaires que nous avons récoltés, de 8 à 12, avec également des différences de 1 ou 2 dents entre les deux ectoproctes d'un même individu. Ce caractère n'est donc pas discriminant pour séparer $P$. umbrosus de $P$. digitatus Gerstaecker, qui possède 10 à 12 dents sur les protubérances (PROST, 1995).

\section{0 - Stenares arenosus Navás, 1924}

Stations et dates de récolte. $-\mathbf{S}: 6 . I V .94,20 \mathrm{~h}, 19$, attirée par la lumière. $\mathbf{F}: 10 . I I I .96,16 \mathrm{~h}, 10$, capturé à la main dans un tas de bois; 7.IV.97, $11 \mathrm{~h}, 1$ \%, capturée sur le tronc d'un karité de $15 \mathrm{~cm}$ de diamètre à $1 \mathrm{~m} 60$ de haut environ; 28.IV.97, $7 \mathrm{~h}, 1 \mathrm{t}$.

Le 15.III.96 vers 17 h 30, à Natiékoura, un individu observé posé sur un arbuste (non capturé). Capturé du 10.III au 28.IV. "Rare" dans la région de Sikasso. Semble inféodé aux zones boisées et, contrairement aux autres espèces, les adultes se posent sur les troncs d'arbres et non sur le sol ou les tiges des graminées.

Les deux mâles de notre collection présentent la plupart des caractères morphologiques de $S$. arenosus décrits par PROST (1995), en particulier la callosité tachée de blanc de la base des ectoproctes, et la forme allongée des ailes; les rapports longueur/largeur, pour les femelles, sont de 4,7 et 5,3 pour les ailes antérieures et postérieures respectivement (fig. 14). Cependant la concavité entre les branches des paramères ne possède pas "quelques épines courtes", comme c'est le cas pour le mâle étudié par PROST (1995), mais 24 soies assez fortes, en particulier les 12 supérieures, qui dépassent en longueur les branches des 


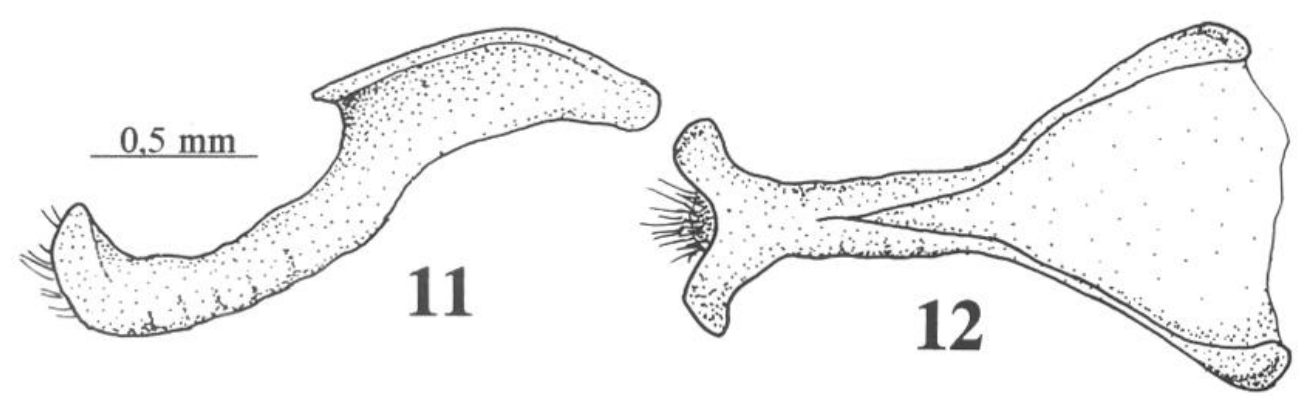

Fig. 11-12. - Stenares arenosus Navás. - 11, Complexe gonarcus-paramères, vue latérale droite. - 12, Idem., de dessous.
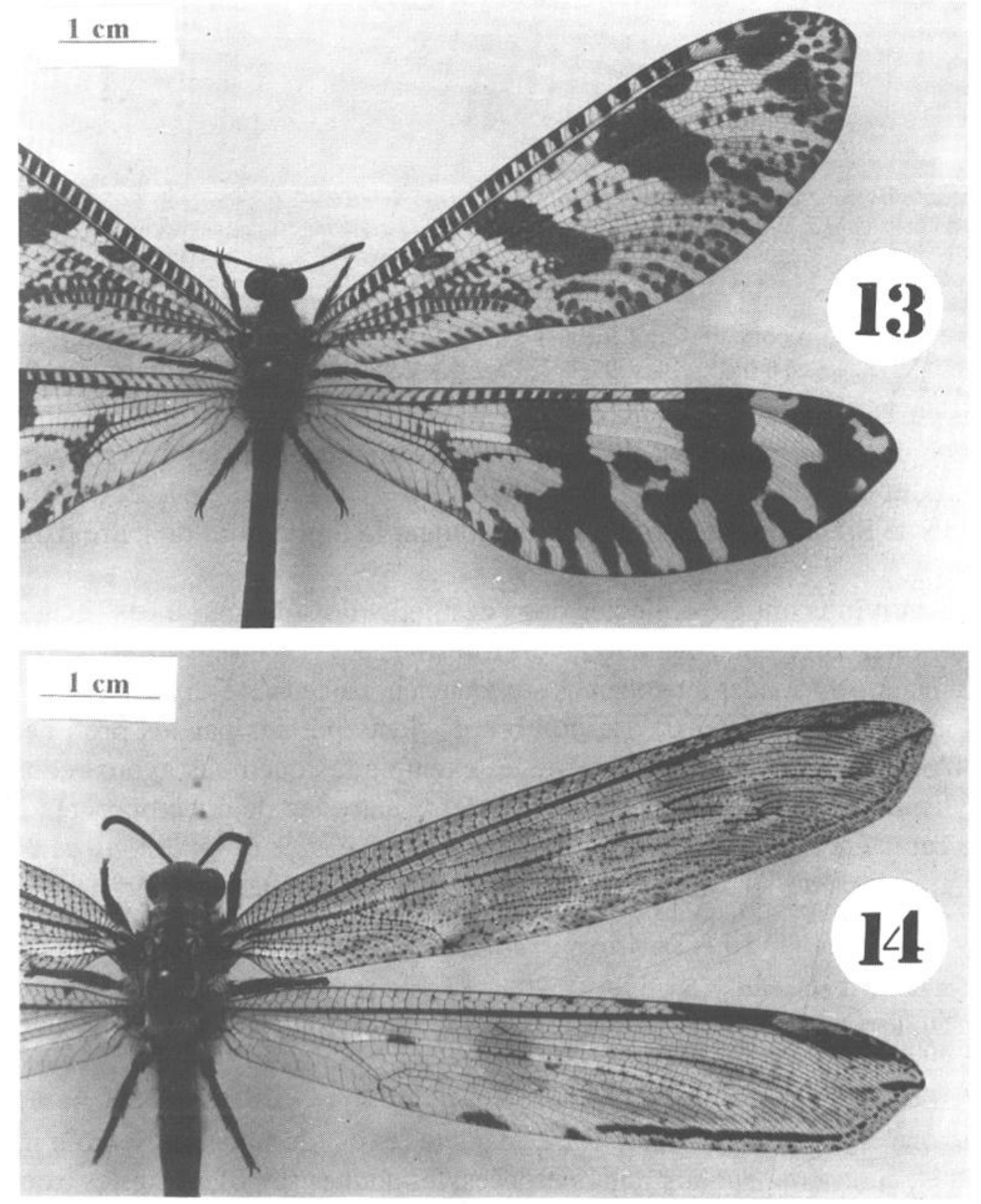

Fig. 13-14. - 13, Palpares umbrosus Kolbe ?. - 14, Stenares arenosus Navás 9.

paramères comme chez $S$. hyaena (fig. 11-12). Il est donc possible que l'individu observé par cet auteur ait été détérioré, ou que la taille et le nombre de ces soies présentent des variations importantes. Ce caractère ne permet donc pas, au moins dans certains cas, de distinguer $S$. arenosus de $S$. hyaena, et seul l'examen de séries plus importantes permettra de trancher. Les individus de Farako ont les ailes relativement sombres. Elles présentent une teinte générale grise, avec trois zones plus claires sur les ailes antérieures (fig. 14). 


\section{1 - Tomatares clavicornis (Latreille, 1830)}

Stations et dates de récolte. - F : 18.XI.97, 1 $9 . \mathbf{S}: 11 . X I .96,19$, capturée le matin posée contre un mur au-dessous d'une lampe. P: 13.X.97, $1 \delta ; 14 . X .95,1 \delta ; 17 . X .97,1 q ; 23 . X .93,1 \delta^{\star} ; 24 . X .93,1 q ;$

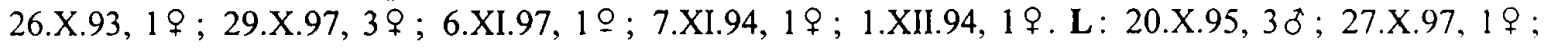

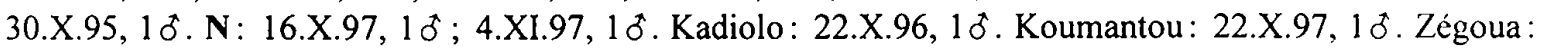

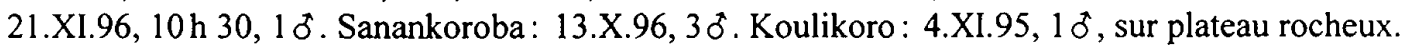

Capturé du 13.X au 1.XII. Très commun dans les graminées.

\section{DisCussion}

Les collectes de Myrmeleontidae que nous avons réalisées dans la région de Sikasso mettent en évidence la richesse spécifique relativement importante de ce groupe dans cette partie du Mali, qui, il faut le souligner, n'avait jamais été prospectée. Nous avons en effet capturé près de 80 espèces différentes, dont la plupart (Myrmeleontinae) n'ont pas encore pu être identifiées. A titre de comparaison, on peut mentionner que MANSELL (1996) estime à environ 180 le nombre d'espèces de Myrmeleontidae présentes en Afrique du Sud, pays au demeurant très étendu, et qui possède de nombreuses éco-régions. Cette biodiversité importante des Myrmeleontidae dans le sud du Mali, se retrouve chez les Palparinae avec onze espèces recensées dans cette note. Si l'on adopte la classification proposée par PROST (1995), outre l'espèce ubiquiste $N$. tigris, au moins une espèce des régions arides ( $P$. radiatus), toutes les espèces de savanes sèches $(P$. spectrum, $P$. furfuraceus, $P$. incommodus, $P$. latipennis,

Tableau I. - Périodes de capture des Palparinae dans les régions de Sikasso et de Koutiala (en noir) et à Bandiagara (en gris) (les chiffres renvoient au texte; $m=$ mâle; $f=$ femelle).

\begin{tabular}{|c|c|c|c|c|c|c|c|c|c|c|c|c|}
\hline \multirow[t]{2}{*}{ esp. } & \multicolumn{12}{|c|}{ mois et décades } \\
\hline & janv. & févr & mars & avril & mai & juin & juill. & aoat & sept. & oct. & nov. & déc. \\
\hline $1 \mathrm{~m}$ & & & & & & & & & & & & \\
\hline $1 \mathrm{f}$ & & & & & & & & & & & & \\
\hline $2 \mathrm{~m}$ & & & & & & & & & & & & \\
\hline $2 f$ & & & & & & & & & & & & \\
\hline $3 \mathrm{~m}$ & & & & & & & & & & & & \\
\hline $3 f$ & & & & & & & & & & & & \\
\hline $4 \mathrm{~m}$ & & & & & & & & & & & & \\
\hline $4 f$ & & & & & & & & & & & & \\
\hline $5 \mathrm{~m}$ & & & & & & & & & & & & \\
\hline $5 f$ & & & & & & & & & & & & \\
\hline $6 \mathrm{~m}$ & & & & & & & & & & & & \\
\hline $6 f$ & & & & & & & & & & & & \\
\hline $7 \mathrm{~m}$ & & & & & & & & & & & & \\
\hline $7 f$ & & & & & & & & & & & & \\
\hline $8 \mathrm{~m}$ & & & & & & & & & & & & \\
\hline $8 f$ & & & & & & & & & & & & \\
\hline $9 \mathrm{~m}$ & & & & & & & & & & & & \\
\hline $9 f$ & & & & & & & & & & & & \\
\hline $10 \mathrm{~m}$ & & & & & & & & & & & & \\
\hline $10 \mathrm{f}$ & & & & & & & & & & & & \\
\hline $11 \mathrm{~m}$ & & & & & & & & & & & & \\
\hline $11 \mathrm{f}$ & & & & & & & & & & & & \\
\hline
\end{tabular}


S. arenosus, $T$. clavicornis), et au moins trois espèces sur quatre de savanes humides ( $P$. nigrescens, $P$. obsoletus, $P$. umbrosus) sont présentes. Parmi ces dernières, qui semblent atteindre ici la limite nord de leur aire de répartition, seule la première est commune. La capture de $P$. umbrosus et $P$. obsoletus porte à 12 le nombre de Palparinae présents au Mali, ce qui en fait le pays d'Afrique de l'Ouest comptabilisant le plus grand nombre d'espèces signalées. $P$. tessellatus, que nous n'avons pas capturé dans la région étudiée, est inféodée aux régions arides sahélienne et désertique.

Cinq espèces volent en fin de saison des pluies et en début de saison sèche: $N$. tigris, $P$. furfuraceus, $P$. latipennis, $P$. obsoletus et $T$. clavicornis. Deux espèces, $P$. nigrescens et $S$. arenosus, sont inféodées à la période la plus sèche de l'année. Enfin, $P$. incommodus, $P$. umbrosus, $P$. spectrum et $P$. radiatus, apparaissent en début de saison des pluies.

Dans la région de Sikasso, aucun Palparinae n'a été capturé de la deuxième décade de juillet à début septembre, c'est-à-dire pendant la période la plus pluvieuse de l'année, et de la fin décembre à la fin janvier où sont enregistrées les températures les plus basses (tableau I).

Pour certaines espèces au moins, l'apparition des mâles semble précéder celle des femelles, et la période de vol de ces dernières paraît se prolonger par rapport à celle des mâles (tableau I).

Enfin, au moins deux espèces, $P$. incommodus et $P$. nigrescens, peuvent être capturées par des oiseaux.

Remerciements. - Nos vifs remerciements au Dr A. Prost pour la relecture du manuscrit et les informations qu'il nous a obligeamment communiquées. Nous remercions également le Dr M. W. Mansell (Plant Protection Research Institute, Pretoria, Afrique du Sud) pour ses suggestions et la correction du résumé en anglais.

\section{AUTEURS CITÉS}

L'Hote Y. \& MAHÉ G., 1996. - Afrique de l'ouest et centrale. Précipitations moyennes annuelles (période 1951-1989). Paris: éditeur ORSTOM.

MANSELl M. W., 1996. - The ant-lions of southern Africa (Neuroptera: Myrmeleontidae). Genus Palparellus Navás, including extralimital species. African Entomology, 4 (2): 239-267.

Prost A., 1991. - Révision de Tomatares clavicornis (Latreille, 1830) (Neuroptera, Myrmeleontidae, Palparinae). Revue française d'Entomologie (N.S.), 13 (4): 145-149.

1995. - Révision des Palparinae d'Afrique de l'Ouest (Neuroptera, Myrmeleontidae). Bulletin de la Société entomologique de France, 100 (1): 79-107.

WHITE F., 1981. - Carte de la végétation de l'Afrique. Paris: UNESCO.

— 1986. - La végétation de l'Afrique. Mémoire accompagnant la carte de végétation de l'Afrique.

Paris : éditeurs ORSTOM/UNESCO, $384 \mathrm{p}$.

Le Bulletin de la Société entomologique de France a édité un NUMÉRO HORS SÉRIE, 100, 1995, 98 pp.

\section{Hymenoptera Apoidea Gallica}

par Pierre RASMONT, P. Andreas EBMER, Józef BANASZAK \& GijS VAN DER ZANDEN

Une liste complète de 913 espèces des Apoidea de la région francophone d'Europe occidentale est présentée. Index de plus de 2800 noms. Bibliographie de 188 références.

Vendu $150 F$ ttc $\quad(100 F$, franco, ttc, aux membres de le S.E.F.) 